\title{
Optimal Environment for the Implantation of Human Embryo
}

\author{
Paweł Kuć \\ Medical University of Biatystok, Department of Perinatology \\ Centre for Reproductive Medicine KRIOBANK, Biatystok, \\ Poland
}

\section{Introduction}

The molecular basis of embryo-maternal relationship, that leads to proper implantation into a receptive maternal endometrium to successfully establish a pregnancy, is still not fully understood. The human endometrium undergoes morphological and hormonal changes during the menstrual cycle in preparation for successful embryo implantation, or menstrual shedding in the absence of implantation. The success of implantation process depends on a receptive endometrium, a blastocyst quality as well as embryo-endometrial interface synchronization. Only $5 \%$ of collected oocytes and only $20-25 \%$ of transferred embryos can lead to a birth of a healthy newborn.

How can we improve implantation rate following infertility treatment? Two main hypotheses can be put forward as a solution:

1. Patient-determined embryo quality,

2. Endometrial quality: patient-determined or a results of a synchronous steroid hormone preparation.

The discussion on these hypotheses will be presented in the following issues of this chapter.

\section{Embryo quality}

Since the in vitro fertilization (IVF) procedure was involved to Assisted Reproductive Technology (ART), there are lots of efforts trying to improve the IVF outcomes. At the beginning, the higher pregnancy rates observed in IVF cycles than in natural, were obtained by stimulating the growth of more than only one follicle as well as by transferring to the uterus excessive numbers of embryos. It has been led to negative side effect as multiple pregnancy, which is nowadays considered as a complication of infertility treatment. The quality of embryos seems to be the basic determinants of success for embryo implantation in the natural as well as in the stimulated cycles. The most important challenge in ART nowadays is the ability to identify the embryos with the greatest development potential. These embryos should be selected and transferred to the uterus.

Some studies have supported the idea that only embryo quality is the best predictive factor for pregnancy in IVF cycles (Brezinova et al.,2009; Fauque et al.,2007), significantly better 
than the assessment of endometrial features (Terriou et al.,2007; Terriou et al.,2001; Zhang et al.,2005). Some of them present results showing the relations between endometrial features and IVF outcomes analysed at different stages of embryo development.

There are lots of methods for evaluating embryo implantation potential and correlations between characteristics of follicles, the oocytes, the zygotes, the early cleavage embryos, the morulas and blastocysts. The ideal method for embryo selection should be non-invasive, easy to assess, standardized, with correlation to pregnancy outcomes.

The studies concerning the determination of new methods for assessing embryos using metabolomics, proteonomics and genomics are still pending, and their results are not yet clinically applicable. Most laboratories still use morphological criteria for embryo assessment. The assessment of embryo morphology is the most important part of many embryo scoring systems. Morphological characteristics provide a lot of information about regular or irregular embryo development, but also present many limitations. They cannot detect genetic disorders of gametes or embryos, and either predict normal pregnancy outcome.

In natural cycles fertilization of oocyte and early cleavage embryo development have place in fallopian tube and then blastocyst is transferred to the uterus where it is implanted in uterine endometrium. In the IVF cycles the early embryo development from zygote to blastocyst can be observed in lab conditions, where the analysis of morphological characteristic is allowed.

\subsection{Zygote scoring systems}

The most important predicting factor of normal embryo development in the zygote stage is the visualization of two pronuclei (PNs), one from sperm cell and one from oocyte. It should occur between 16 and 19 hour after fertilization. In the past, 5 grade zygote scoring system presented by Scott et al. (Scott et al.,2000) was based on five zygote features: PNs size, the nuclear alignment, the nucleolar alignment and the distributions, and the position. Grade 1 was described as equal number of nucleoli aligned at the pronuclear junction. Grade 2 - as an equal number of nucleoli in equal size in the same nuclei, and one of nuclei having alignment at the pronuclear junction, and the second one with scattered nucleoli. Grade 3 was described as equal numbers of nucleoli in equal size equally scattered in the two nuclei. Grade 4 - unequal numbers of nucleoli and/or unequal size. Grade 5 - with not aligned pronuclei, in different size, and not placed in the central part of zygote. This system was then modified by author to the 4 grades $\mathrm{Z}$ score system. The following parameters which were assessed: PNs size and alignment, aligment of nucleoli within the PNs and cytoplasmic morphology. The total number of points which could be achieved was ranged between 3 and 35. Embryos with more than 15 points were classified as embryos with the highest implantation potential, and presented about 70\% pregnancy rate (Scott et al.,2000; Scott \& Smith,1998).

The last modification of Z score system was proposed in 2003 by Scott $(S \operatorname{cott}, 2003)$. The scoring system was based on the zygotes features that can be observed at the time of fertilization. The last version has been commonly used by many labs. In comparison to the previous version the presence of halo was associated with better morphology of embryos assessed in the $3^{\text {rd }}$ and the $5^{\text {th }}$ day of their development. Zygotes assessed as Z1 and Z2 had 
the highest implantation rate. The Z1 and Z2 zygotes presented significantly higher number of good quality blastocysts on the $5^{\text {th }}$ day after fertilization. All pregnancies from day 5 transfers resulted from the transfer of blastocysts that originated from Z1 or Z2 zygotes. Figure 1 shows comparison the 5 grade score with $\mathrm{Z}$ zygote scoring system.

\begin{tabular}{ll}
$\begin{array}{c}\text { 5-grade score } \\
\text { system }\end{array}$ & $\mathrm{Z}$ score \\
\hline Grade 1 & $\mathrm{Z} 1$ \\
morpholear \\
Grade 2
\end{tabular}

Fig. 1. The 5 grade score and $\mathrm{Z}$ zygote scoring systems.

There are some studies that evaluate the usefulness of cumulative zygote scoring systems on embryo implantation. Ludwig et al. (Ludwig et al.,2000) presented modified Scott's Z score system, where the threshold for positive IVF results was 13 points. Embryos presented less than 13 points according to the $Z$ score system presented negative implantation results in 92\%. Zollner et al. (Zollner et al.,2002) created the cumulative zygote scoring system similar to the $\mathrm{Z}$ score system. The following features of zygote were used to evaluate zygote quality: 
number, the position and size of pronuclei, the alignment and the number of nucleoli in each pronuclei, halo effect, morphology of vacuoles and structure of cytoplasm. The zygotes with more than 15 points were associated with higher quality of blastocyst in the $5^{\text {th }}$ day of embryo development.

Another zygote scoring system was proposed by Tesarik and Greco (Tesarik \& Greco,1999). They created the system based on the assessment of the number and distribution of each PN. The system divide embryos into 2 groups: pattern 0 and non pattern 0 . Its allows efficient prediction of implantation rate using static, single observation of zygote's state. Authors reported $30 \%$ implantation rate among pattern 0 embryos, and $11 \%$ among non pattern 0 embryos.

The assessment of zygote morphology at 16 and 19 hour after fertilization seems to be helpful in selection of the embryos with the highest potential for implantation. The evaluation of zygote morphology seems to be helpful in countries with internal law regulations imposing embryo selection at the PNs stage. This scoring systems can be used in patients who would have benefited from the $5^{\text {th }}$ day embryo transfer (ET), especially in countries where the high costs of ART techniques exist parallel with the poor, not optimal embryo culture systems.

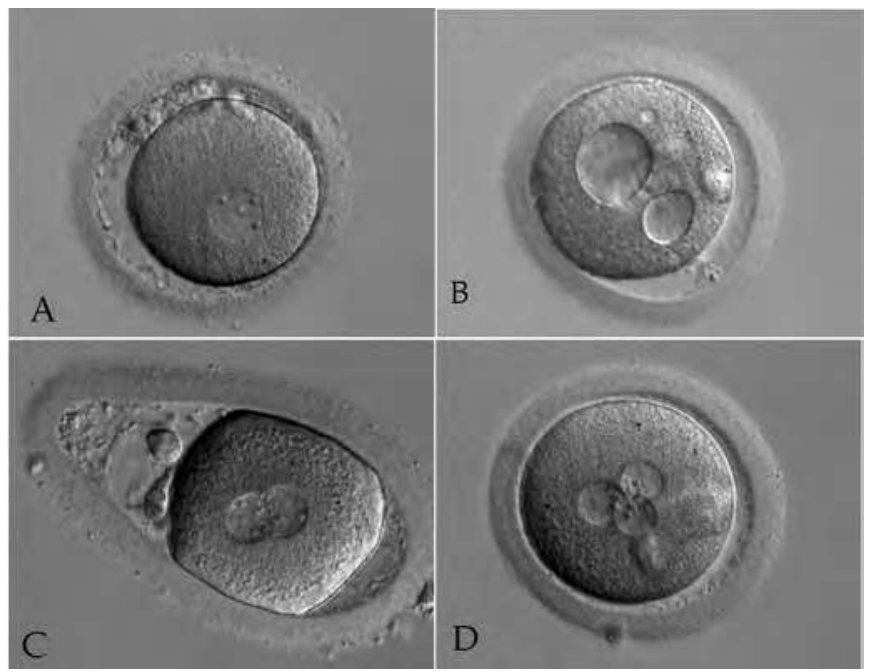

Fig. 2. Abnormalities of zygote stage embryos: A - mononucleation (1PN); B - vacuoles in cytoplasm; C - dysmorphic zygote (2PNs); D - multinucleation (3PNs).

\subsection{Cleavage embryos scoring systems}

Twenty five hours after the fertilization embryos reach 2-cell stage of their development. There are very few studies focusing on this stage and on its impact on the positive implantation. Most of them (Ciray,2007; Ciray et al.,2004) presented the conclusions that assessment of 2-cell embryo stage together with the subsequent stages of embryo development can present additive positive value in embryo reproductive potential and implantation rate. 


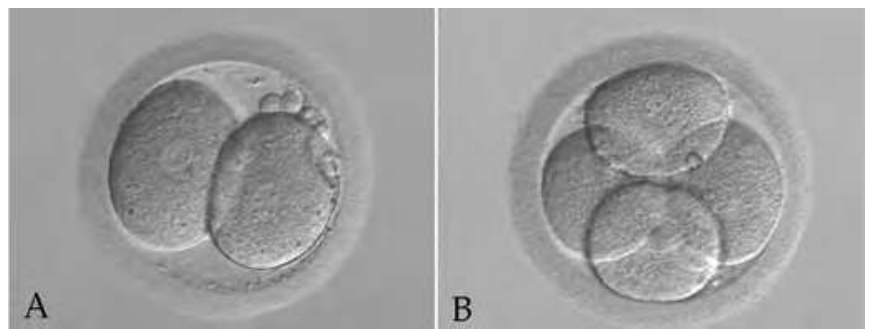

Fig. 3. Good quality early cleavage embryos: A - 2 blastomer stage; B - 4 blastomer stage.

The perfect 4-cell embryo should have equal size of regular symmetrical blastomers with minimal fragmentation (Giorgetti et al.,2007; Terriou et al.,2007). Nowadays, the nucleation of blastomers has important value in selection of abnormal embryos. Multinucleated blastomers are associated with lower implantation rate due to the high risk of chromosomal abnormalities. Mononucleation of all 4 blastomers is associated with higher embryo reproductive potential (Saldeen \& Sundstrom,2005).

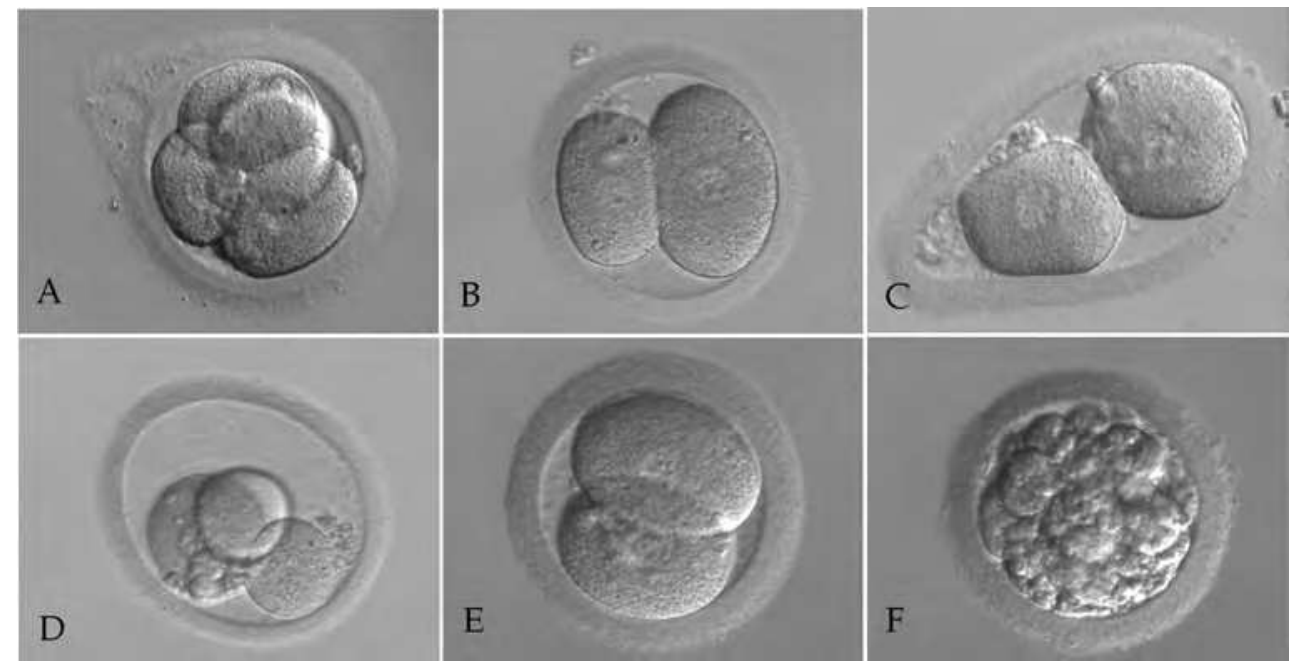

Fig. 4. Abnormalities of early cleavage embryos: A - defect of zona pellucida; B - irregular size of blastomers; C - dysmorphic 2 blastomer embryo; D - shrink blastomers; E - thick zona pellucida; $\mathrm{F}$ - total cytoplasm defragmentation.

On the third day after fertilization 8-cell stage of embryo development should be confirmed. The assessment of three day embryo includes the number of cells, the symmetry of blastomers, the degree of fragmentation, and eventually the early embryo compaction. Many studies confirmed the relationship between the number of blastomers observed on the third day after fertilization and implantation rate following transfer taking place on this day. Some authors suggest that embryos having exactly 8 blastomers on the third day present the highest reproductive potential. These embryos have the highest implantation rate (Racowsky et al.,2003). 


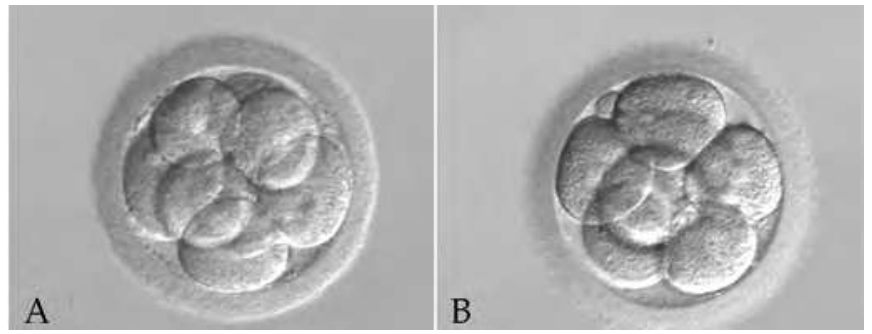

Fig. 5. Good quality cleavage stage: A - 8 blastomer stage; B - 6 blastomer stage.

Another study showed that at least 8 or more blastomers observed in the third day of embryo development were associated with higher implantation rate in comparison to less than 8 cells (Carrillo et al.,1998). Other authors maintained that 7 to 9 blastomers observed on the third day was connected with the highest implantation potential (Alikani et al.,2000).

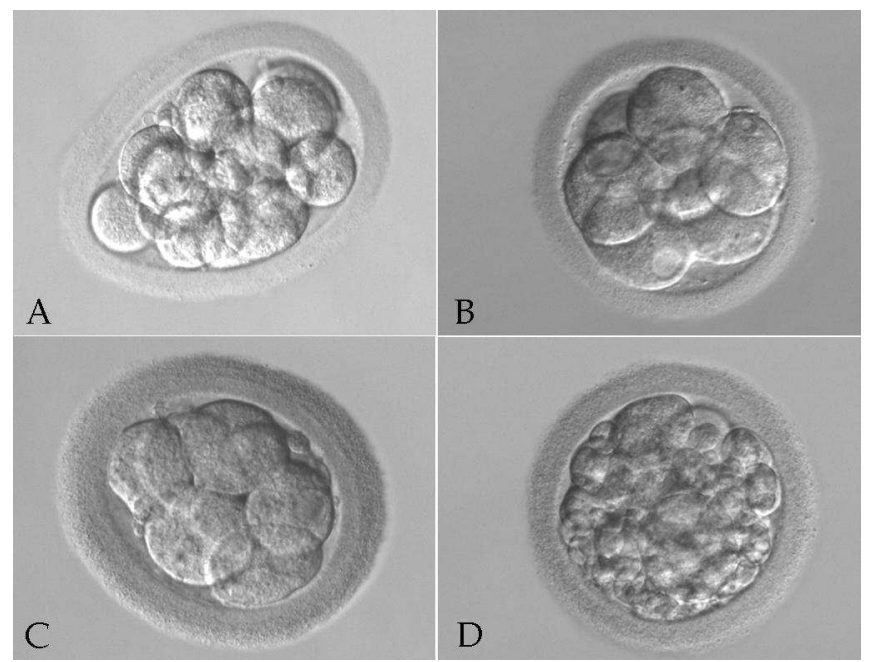

Fig. 6. Abnormalities of cleavage embryos: A - dysmorphic embryo; B - vacuole into one of blastomers; C - thick zona pellucida; D - total cytoplasm defragmentation.

The percentage of fragmentation is still under discussion by many authors. The minimal fragmentation and symmetry of blastomers positively correlate with implantation rate similar to the 4-cell stage embryos. There are few studies that present that the early visualization of embryo compaction on the third day of embryo development is a positive prognostic factor for further embryo implantation. In at least 8-blastomer embryos with minimal fragmentation, early compaction was associated with higher implantation rate (Skiadas et al.,2006). Desai et al. (Desai et al.,2000) suggested that to assess reproductive potential of 3 day embryo, the following features should be taken under consideration: number, size and the symmetry of blastomers; compaction; the grade of fragmentation; vacuoles in cytoplasm; blastomer expansion. The evaluation of this characteristics was connected with more than $80 \%$ sensitivity in prediction of positive implantation. 


\subsection{Blastocyst scoring systems}

The progress in ART techniques, especially in culture media and metabolimics, allow longer incubation of embryos in lab conditions and their development to blastocyst stage. The transfer of blastocyst imitates the natural conception cycles, where such "embryo-uterine dialogue" is responsible for creation of a new life. Similar to the cleavage stage embryos, embryo morphological features are very important in assessment of blastocyst quality and its reproductive potential. Most of blastocyst scoring systems are based on evaluation embryo morphology and are focused on: blastocyst development stage - expansion and hatching status, inner cell mass (ICM) quality; trophoectoderm (TE) quality. The blastocyst stage is usually reached on the fifth day of embryo development. One of the most common used blastocyst scoring system was proposed by Gardner (Gardner et al.,2000).

Blastocysts are named using numerical score from 1 to 6 according to their expansion and hatching status:

1. Early blastocyst, blastocoel cavity less than half the volume of the embryo

2. Blastocoel cavity more than half the volume of the embryo

3. Full blastocyst, cavity completely fills the embryo

4. Expanded blastocyst, cavity larger than the embryo, with thinning of the zona

5. Hatching blastocyst, the trophoectoderm has started to harniate through the zona

6. Hatched blastocyst, has completely escaped from the zona

The blastocyst are also assessed according to ICM quality:

a. Many cells, tightly packed

b. Several cells, loosely grouped

c. Very few cells

As well as according to TE quality:

a. Many cells, forming a cohesive layer

b. Few cells, forming a loose epithelium

c. Very few large cells

Blastocysts are given a quality grade for each of the 3 components and the score is expressed with the expansion grade listed first, the inner cell mass grade listed second and the trophectoderm grade third. For example, a blastocyst quality grade of 4AA means that the blastocoel cavity is expanding and the zona is thinning (grade 4), it has many tightly packed cells in the inner cell mass (grade A), and has a trophectoderm can be seen to be composed of numerous cells (grade A).

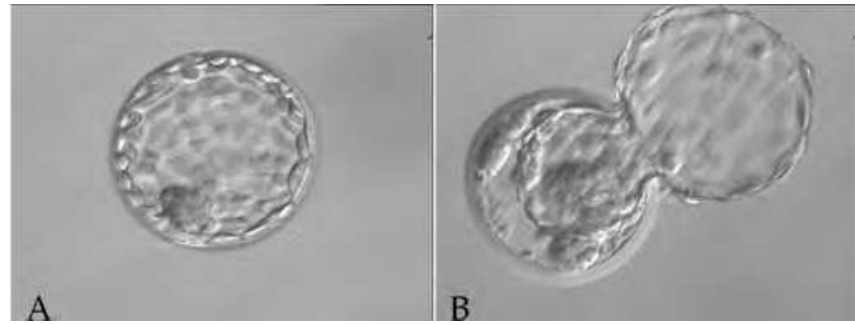

Fig. 7. Good quality blastocysts: A - good quality expanded blastocyst (4AA); B - hatching blastocyst (5AA). 
Another scoring system was proposed by Dokras et al. (Dokras et al.,1993). The blastocyst grading according to following schema is presented as: grade 1 blastocysts with early cavitation resulting in the formation of an eccentric and then expanded cavity lined by a distinct ICM region and TE layer; grade 2 blastocyst exhibited a transitional phase where single or multiple vacuoles were seen that over subsequent days developed into the typical blastocyst appearance of the grade 1 blastocysts; grade 3 - blastocysts with several degenerative foci in the ICM with necrotic cells. The Dokras system is mainly based on embryo developmental potential and expansion of the blastoceol cavity. The Gardner's system is more detailed than Dokras system, taking into account the appearance of ICM and TE quality. Balaban et al. (Balaban et al., 2006) in randomized trial compared both scoring systems, and observed the better outcome in the Gardner system, where higher implantation rates were observed. Gardner's system offers higher selective criteria for the blastocysts, which in turn is reflected in the clinical outcome following embryo transfer.

Rehman et al. (Rehman et al., 2007) presented a numerical blastocyst-morphology grading system (blastocyst quality score BQS). This system correlate with criteria established by Gardner. BQS is defined as the product of degree of expansion and hatching status and ICM and TE grades, where letter grade $A$ is given the value 3 , grade $B$-value 2 , and grade $C$ value 1. For example BQS calculation for a $4 \mathrm{AB}$ blastocyst is $4 \times 3 \times 2$, with a BQS of 24 points. The authors calculate each Gardner's scale score with BQS. Authors tried to present a possible correlation between BQS and number of cells in the blastocysts, but they did not present the number of cells in studied blastocysts. Although Gardner's blastocyst morphology grading system is clinically useful scheme and has been shown to predict implantation rates, there are several limitations associated with the use of BQS in clinical practice.

In the literature, there are few studies investigating the usefulness of cumulative, combined multiday embryo scoring systems in evaluation the embryo reproductive potential. The investigators tried to combine morphological characteristics of zygotes, cleavage stage embryos and blastocysts to improve the embryo selection process. Available data suggests that combined mulitiday embryo assessment provides many usefulness information about embryo development, than a single day scoring systems. However, there is no ideal multiday system which could be implemented to clinical practice nowadays. There have been still undetectable critical time points of embryo development which could provide accurate information about the ideal time for successful embryo transfer.

More clinical trials are needed to find more accurate embryo selection parameters. Over the last few years, few continuous time lapse embryo monitoring systems were presented, as a device that can improve IVF outcomes by better embryo selection. Special incubators equipped with digital camera allows 24 hours observation of embryo incubation, from the conception till the time of transfer. Images of each embryo are automatically recorded at preset time intervals. Detailed information about timing of cell divisions and other critical events can provide future extensive documentation that embryologists can use to select a viable embryo for transfer during an IVF treatment. This method is reported as sufficiently safe. Constant embryo monitoring is performed inside incubators, so the embryos are never exposed to adverse growth conditions or temperatures. Images and detailed information about incubation conditions can be stored in patient data files for future reference. There is also an global idea for creation of the large database of embryo images (more than 20 million images) and a 
detailed analysis of the development pattern of embryos that successfully implanted and resulted in ongoing clinical pregnancies compared to embryos that failed to implant. The prospective clinical trials are required to analyze the obtained data, which can contribute to creation of novel embryo selection procedures, and thus improve IVF outcomes.

The optimum day for embryo transfer procedure seems to be established. Most of the recent studies suggest that the ideal time for embryo transfer is blastocyst stage in the fifth day. Prolonged embryo culture and the availability of blastocysts for transfer are associated with increased implantation rate and better neonatal outcomes compared with 2 or 3 day transfers of cleavage embryo stages (Blake et al.,2007; Kallen et al.,2010; Gardner et al.,2000; Papanikolaou et al.,2006). Taking under consideration the high implantation rate of blastocyst, a single embryo transfer can be offered for selected patients, which tends to reduce the number of multiple pregnancies (Kawachiya et al.,2011; Papanikolaou et al.,2010). The transfer only one blastocyst in the first attempt may lead to higher cumulative pregnancy rate by allowing transfer remaining frozen blastocyst in the next cycle. However, blastocyst transfer may not be ideal for all IVF patients, in all clinical situations. A Cochrane review of 16 trials on efficiency of blastocyst and day 3 embryo transfer, did not find the significant differences in pregnancy rate, miscarriages rate and multiple pregnancy. Some IVF canters still have problems with blastocyst culturing and/or freezing so the third day of transfer is an optimal solution in these cases. The problem of the optimal day of embryo transfer return back nowadays, and remains a significant element of discussion in many scientific societies working on reproductive medicine.

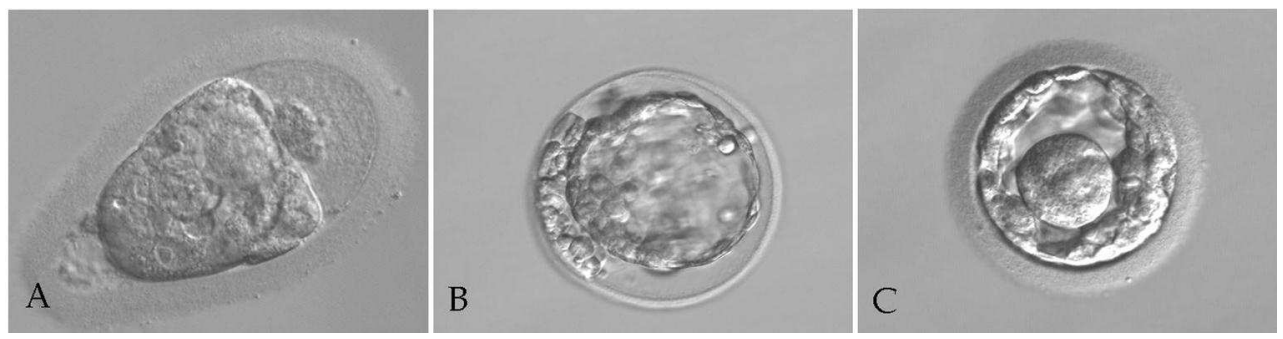

Fig. 8. Abnormalities of 5 day embryos: A - dysmorphic morula; B - degenerating blastocyst before hatching; $\mathrm{C}$ - remained undeveloped blastomer in blastocyst.

The assessment of just the embryo morphology cannot disclose, whether a particular embryo will be implanted or not, and it can not disclose all the information contained in the embryo. The most precise evaluation of the impact of embryo quality and endometrial features on the pregnancy rate should be carried out on patients with a single, high quality blastocyst transfer. The analysis of all the information obtained from the elective single embryo transfer procedures can help embryologist in the better embryo selection, leading to the improvement of implantation and pregnancy rates. The role of a single embryo transfer, the limitations of multiple embryo transfer, and the role of cryopreservation are still discussed nowadays.

\section{Growth factors and endometrial receptivity}

The estrogen dependent proliferative phase of the endometrium, which lasts from the menstrual bleeding until ovulation, is characterized by proliferation of endometrial glands 
and endometrial stromal tissues as well as neoangiogenesis of endometrial blood vessels. The progesterone dependent secretory phase, which starts after ovulation with a peak of luteinizing hormone ( $\mathrm{LH}$ ) and ends with menstrual bleeding, is accompanied by high secretory activity of endometrial glands, elongation and growth of spiral arteries as well as decidualization of endometrial stroma. These all mechanisms enable embryonic invasion during the implantation window. Growth factors, cytokines and chemokines play an important role in paracrine and autocrine actions to affect the histologic and biochemical changes in endometrium before and during the implantation process.

Communication between the embryonic and maternal tissues, while the embryo is transferred through the fallopian tube and in uterine cavity, seems to be an important factor in the successful embryo implantation. The embryo communicate with the maternal organism, by producing and secreting several cytokines, chemokines, growth factors during its development.

At the time of implantation, according to increase the production of prostaglandins (mainly prostaglandin $E_{2}$ ) in endometrium, the increase of capillary permeability in endometrial stroma is observed. By this time, secretory activity presents a peak, and endometrial cells becoming to be reach in lipids and glycogen. The window for endometrial receptivity is restricted to the $20^{\text {th }}-24^{\text {th }}$ day in spontaneous 28 -day regular cycle. Progesterone-induced formation of pinopodes (surface epithelial cells with smooth protrusions) appears on the endometrial surface. The pinopodes are able to absorb an intrauterine fluid trying to bring the blastocyst closer to their surface. Pinopodes appear around the $21^{\text {st }}$ day of the cycle and exist only for a few days. After hatching the blastocyst from the zona pellucida, blastocyst adheres to the endometrial surface. During the last decade, the knowledge about communication between implanting blastocyst and maternal endometrium increased due to novel RNA and protein microarrays analyses. Without the presence of blastocyst, decidua synthesizes the factors that inhibiting the implantation process such: insulin-like growth factor binding protein-1 (IGFBP-1), tissue inhibitors of metalloproteinases (TIMPs). On the other site blastocyst promotes secretion the following factors counteracting maternal restraint mechanisms and promote embryo implantation into the endometrium: matrix metalloproteinases (MMPs), leukaemia inhibitory factor (LIF), epidermal growth factor (EGF), insulin like growth factor II (IGF II), and interleukins (IL) (Giudice,2003). Trophoblast penetrates the uterine epithelial basement membrane by with use of some proteolytic enzymes, and finally implant in the endometrial stroma. Matrix metalloproteinases (MMPs) are the most important enzymes taking part in endometrial tissue reconstitution during implantation. MMPs degrade the extracellular matrix components as collagens, proteoglycans, glycoproteins. Among all the metelloproteinases, MMP-2 and MMP-9 seems to play the most important role in the tissue remodeling that accompanies implantation and decidualization. MMP-2 may participate in the early phase of decidual remodelling as well as in neoangiogenesis. MMP-9 can coordinate trophoblast invasion into the endometrial layer. Changes in the expression of these two metelloproteinases during implantation can occur without direct signals from the blastocyst. Some investigators indicate that the expression of these two metelloproteinases might be induced by some cytokines or growth factors, such as vascular endothelial growth factor (VEGF), transforming growth factor (TGF), epidermal growth factor (EGF) as well as by their specific tissue inhibitors (TIMPs) (Zhang et al.,2005). 
There are studies suggesting the role of VEGF in endometrial neoangiogenesis during embryo implantation. Angiogenesis is a key process in preparation of endometrium for implantation. VEGF is a specific mitogen for endothelial cells that exerts its functions through two receptor tyrosine kinases (Flt-1 and Flk-1/KDR). These receptors were found in epithelial cells and stroma of endometrium. Neuropilin-1 (NRP-1), another receptor for VEGF, was found overexpressed in endometrium in the proliferative phase, in comparison to secretory phase of the cycle. Charnock-Jones et al. (Charnock-Jones et al.,1994) demonstrated that mRNA VEGF receptor, Flt-1, was overeexpressed in the cytotrophoblast and also by the extravillous trophoblast in the maternal deciduas in early pregnancy. VEGF may induce vascular and endometrial cells proliferation and differentiation, as well as be involved in the invasion process of embryos implanting into the endometrium.

\begin{tabular}{|c|c|}
\hline Cytokines/ Growth factors & Enzymes/Tissue mediators \\
\hline $\begin{array}{l}\text { VEGF - Vascular Endothelial Growth Factor } \\
\text { EGF - Epidermal Growth Factor } \\
\text { IGF I and II - Insulin-like Growth Factor I and II } \\
\text { FGF - Fibroblast Growth Factor } \\
\text { TGF a and } \beta \text { - Transforming Growth Factor a and } \beta \\
\text { Il-1 - Interleukin } 1 \\
\text { Il-6 - Interleukin } 6 \\
\text { Il-11 - Interleukin } 11 \\
\text { LIF - Leukemia Inhibitory Factor } \\
\text { TNF a - Tumor Necrosis Factor a } \\
\text { IGFBP-1 - Insulin-like Growth Factor binding protein-1 }\end{array}$ & $\begin{array}{l}\text { MMP-2 - Metalloproteinase-2 } \\
\text { MMP-9 - Metalloproteinase-9 } \\
\text { TIMPs -Tissue Inhibitors of } \\
\text { Metalloproteinases } \\
\text { PGE }_{2} \text { - Prostaglandin E }\end{array}$ \\
\hline
\end{tabular}

Table 1. Biochemical factors involved in the human embryo implantation.

Interleukins may also exert an influence on human embryo implantation. The increase of the expression of interleukin-1 in endothelial cells of uterine spiral vessels and endometrial stroma was observed before implantation. Decidualization of endometrial stromal cells in vitro is enhanced by interleukin 11. Interleukins can exert an influence on proper endometrial receptivity and could have an important impact on successful semi-allograft blastocyst implantation (Paiva et al.,2007; White et al., 2007). The expression of colony stimulating factor-1 and leukemia-inhibitory factor (LIF) were also found in human endometrium as well as in blastocyst structures. It suggest also their possible role in human embryo implantation (Simon et al.,1996).

Cytokines, chemokines, and growth factors have been still investigated in all tissues associated with implantation as well as in embryonic structures. These factors are the biochemical tools involved in the implantation process. Their cataloguing process is still pending, and their role in consecutive parts of embryo implantation process is still not completely understood. Table 1 presents the most important factors involved in the human embryo implantation.

\section{The role of uterine contractility in embryo implantation}

What could be the reason of recurrent implantation failure among especially young women undergoing IVF treatment, when good quality blastocysts are transferred, in patients with 
any uterine anatomical anomalies, and with any hormonal disorders? The ideal intrauterine conditions that enable implantation include appropriate endometrial status, sufficient endometrial blood perfusion and absence of excessive uterine contractions. The contractile activity of the pregnant and non-pregnant uterus plays an important role in the human reproduction. The stimulation of uterine contractions by estrogens during the follicular phase in the non-pregnant uterus, supports transport of spermatozoas from vagina to the Fallopian tubes. The absence of the uterine contractility, in response to rising progesterone serum level, provides an optimal environment for successful embryo implantation.

Embryo attachment to the endometrial layer and its invasion require the uterine quiescence for implantation (Bulletti \& de Ziegler,2005,2006). Implantation failures in IVF cycles when good quality embryos are transferred, could be a results of increased uterine contractile activity during and after transfer procedure (Fanchin et al.,1998,2001). About 15\% of embryos could be found in vagina after the transfer procedure, and only $45 \%$ of transferred embryos could be found in the uterus one hour after the transfer (Poindexter et al.,1986).

Implantation rate seems to be strictly dependent on the uterine activity during embryo transfer. The excessive uterine contractile activity during ET procedure is found in about $30 \%$ of IVF patients. Some investigators found almost 3 times higher clinical pregnancy rates in patients with uterine quiescence, than in women with excessive uterine activity (53\% and 13\%, respectively) (Ayoubi et al.,2003). Ayoubi et al. observed changes in uterine contractile activity appearing after ovulation in the spontaneous menstrual cycles and in stimulated IVF cycles. Uterine contractile frequency did not differ between studied groups of patients on the day of luteinizing hormone surge ( 5 uterine contractile per 1 minute) and hCG administration (5.3 uterine contractile per $1 \mathrm{~min}$ ). In the normal regular menstrual cycle, the uterine quiescence is reached about 4 days after the peak of luteinizing hormone. Uterine contractile activity in IVF cycles remained elevated until the $4^{\text {th }}$ day after hCG administration, despite of higher serum progesterone levels reached after additive oral or vaginal progesterone supplementation. The limit of uterine contractility after exposure to vaginal progesterone was established by Fanchin et al. study (Fanchin et al.,2001) and Bulletti et al study (Bulletti \& de Ziegler,2006). Seven or less contractions detected during five minute intervals in ultrasound measurements were found as uterine quiescence. More than 7 contractions detected during five minute intervals were presented as increased uterine activity. This excessive uterine activity can be also amplify in stimulated cycles, by higher serum concentration of estrogens stimulating uterine contractility. In IVF patients uterine activity has declined on the $6^{\text {th }}$ day after human chorionic gonadotropin $(\mathrm{hCG})$ administration (Ayoubi et al.,2003). These findings can be explanation to improved implantation rate observed after blastocyst transfers. There are many studies confirming the highest implantation rate after the transfer of embryos in blastocyst stage (Fauque et al.,2007; Zander-Fox et al.,2011; Kallen et al.,2010; Gardner et al.,2000). If the uterine contractile frequency decreases at the time of blastocyst transfer in patients with excessively expressed uterine activity, the postponing of the embryo transfer procedure could be considered in these group of patients during IVF treatment.

The study by Fanchin et al. (Fanchin et al.,2001) showed the possible influence of decreased uterine contractility on the high blastocyst implantation rate. Investigators compared the frequency of uterine contractions on the day of hCG administration, on the fourth day after hCG and on the 7th days after hCG. The significant decrease in uterine contractions frequency 
was observed from the day of hCG administration (4.4 uterine contractions per 1 minute), by the $4^{\text {th }}$ day after hCG (3.5 uterine contractions per 1 minute), to the $7^{\text {th }}$ day after hCG (1.5 uterine contractions per 1 minute). The decrease of uterine activity was progressive, and reached the lowest frequency on the $7^{\text {th }}$ day, at the time of blastocyst transfers. This uterine quiescence can be associated with the higher blastocyst implantation rate, in comparison to cleavage embryos. However, the most of embryos are still transferred on the $2^{\text {nd }}$ or the $3^{\text {rd }}$ day after fertilization, when the elevated uterine contractile activity is observed.

There is no still an ideal method for assessing the uterine activity of non-pregnant uterus. Cardiotocography, bioimpedance measurement or electromyography, or magnetic resonance scanning are restricted to evaluation of the activity of only pregnant uterus. Only few methods assessing the uterine activity in non-pregnant uterus were described in the literature (Kitlas et al.,2009; Rabotti et al.,2009; Kissler et al.,2004). One of the most sensitive method, but unfortunately invasive, is the measurement of changes in intrauterine pressure which reflect the uterine contractile activity.

Kissler et al. (Kissler et al.,2004) tried to evaluate the activity of non-pregnant uterus using hysterosalpingoscintigraphy (HSSG). This study was the first one to be designed to investigate utero-tubal transport function by HSSG and uterine contractility by intrauterine pressure (IUP) measurement. Investigators compared these parameters in 21 patients on the same day in the periovulatory phase. In HSSG, the transport function was visualized using 99m-technetium-marked albumin. In IUP measurement methods, the amplitude and frequency of uterine contractions per minute, as well as the basal pressure tone were assessed by an intrauterine catheter. In periovulatory phase, the mean value of uterine contractions was 3.4 contractions per minute, the mean amplitude was $12.0 \mathrm{mmHg}$ and basal pressure was $70.7 \mathrm{mmHg}$. These two methods positively correlated with uterine activity. The IUP measurement using special catheter or hysterosalpingoscintigraphy are unfortunately invasive and with many limitations in clinical practice.

Kitlas et al. (Kitlas et al.,2009) tried to assess synchronization between contractions in different topographic regions of the uterus in patients with dysmenorrhea. The authors presented four different analytic methods to assess uterine signals: the cross-correlation function, the coherence function, the wavelet cross-correlation and the wavelet coherence. Spontaneous uterine activity was recorded directly by a dual micro-tip catheter consisted of two ultra-miniature pressure sensors. One sensor was placed in the fundus, the other one in the cervix. The study showed, that the analysis of synchronization of the uterine contractions signals may have a diagnostic value, but this method has never been used to assess uterine contractility before embryo transfer.

A promising technique for uterine contractile activity evaluation could be electrohysterography (EHG). The EHG measures the electrical activity from the abdominal surface, which triggers the contraction of the uterine muscle. Rabotti et al. studies (Rabotti et al.,2009) tried to compare electrohysterography with the measurement of intrauterine pressure in pregnant uterus. However, a quantitative estimation of the internal uterine pressure by EHG is not available for clinical practice in reproductive medicine, probably because of small size of non-pregnant uterus.

In humans, ethical issues do not allow to use any invasive assessment of uterine contractions such as intrauterine pressure measurements, during or after embryo transfer 
procedure. Consequently, the assessment of uterine activity should be non-invasive. Several methods involving transvaginal ultrasonography for assessing endometrial and myometrial layer movements have been already described.

The oldest method for assessing the ultrasound images, which can provide information about uterine activity, is the M-mode presentation ultrasound technique (Figure 9). This method allows the assessment of low-level image data and thus does not provide additional information about the object's shape or location. Application is limited by a number of conditions specific to medical imaging. The M-mode technique has a number of disadvantages. This method does not measure the movements of the whole uterus, only the upper and lower boundaries present within a user specified intersection. This lack of provision becomes significant in cases of exaggerated bowel or respiratory movement that may change the location of previously marked gaps in the uterus. The uterus can also move forward or backward in relation to the intersection that has been set. Since the method does not take into account the whole uterine shape into account, only the boundaries crossing the intersection are trackable which can make measurement difficult. Even in ideal conditions where the boundaries are clear and easy to track, accurate measurement can still be difficult to perform. Similarly, it is also difficult to interpret images where contractility is present, but not causing movements of boundaries and influencing only the texture of the endometrium. This technique also depends on proper visualization of the uterus which is highly variable and influenced by factors such as its retrovert position or filling of the urinary bladder.
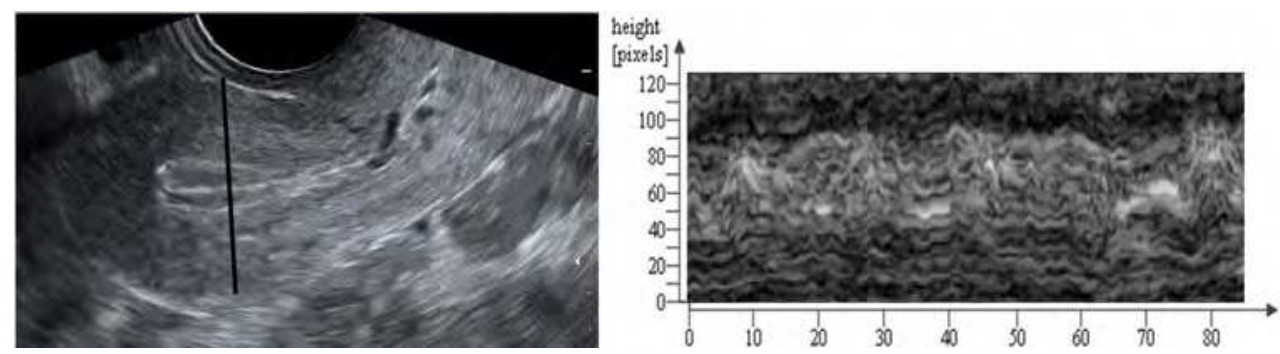

Fig. 9. M-mode ultrasound technique for assessing endometrial and myometrial movements.

Fanchin et al. (Fanchin et al.,1998) proposed a method based on analyzing of a line segment of the uterus and video sequence that shows two-dimensional plot using successive frames. The horizontal component represents line segment length and the vertical component time. Although the method is simple and easy to implement, the prototype clearly demonstrates drawbacks. In the presence of a slight increase in the amount of noise or movement of the uterus, the method is prone to generating mistakes and does not provide useful information about uterine contractility. The method was presented in 1998, and the authors did not analyzed any good quality film sequences, which are available now thanks to the progress in ultrasound imagining. The method was not found to be an accurate tool for the measurement of contractility of non-pregnant uterus.

There are also some possibilities of pharmacological treatment of excessive uterine activity in IVF patients. Oral or vaginal progesterone supplementation, even when acting on uterine receptivity, improving endometrial status, presents limited benefits for implantation rates in patients with excessive uterine activity. Studies assessing the effectiveness of prostaglandin 
synthesis inhibitors and $\beta_{2}$-adrenoreceptor agonists have shown a positive effect on pregnancy rates, but these drugs have failed to enter routine clinical use because of safety concerns.

In randomized control trial, Bernabeu et al. (Bernabeu et al.,2006) analyzed the influence of administration of indomethacin on implantation rate in oocyte donation recipients. Indomethacin is one of the non-steroid anti-inflammatory drugs (NSAIDs), which inhibit the prostaglandin synthesis. Prostaglandins are synthesized from arachidonic acid by cyclooxygenase (COX). Indomethacin blocks the COX enzyme, inhibits the production of prostaglandins and reduces or inhibits the uterine contractile activity. Prostaglandins are the most important factors stimulating the uterine contractions in non-pregnant uterus. Bernabeu et al. (Bernabeu et al., 2006). Oocyte donation is the best model to evaluate the determinants of implantation, because of the limitations concerned with the fertilized oocyte quality. In the studied group of patients the authors administered $100 \mathrm{mg}$ of indomethacin rectally (in three doses every 12 hours) starting on the night prior to transfer. Implantation rate was $27.8 \%$ in the indomethacin group, and $26.4 \%$ in the placebo control group. The study did not show significant differences in implantation rate among studied groups of patients.

Moon et al. (Moon et al.,2004) examined the effect of other prostaglandin synthesis inhibitor piroxicam on implantation rate in IFV patients. In prospective, randomized double-blind, placebo controlled trial the authors compared the effect of piroxicam administered in the day of embryo transfer in patients undergoing fresh as well frozen IVF cycles, on implantation rate. The piroxicam was chosen from several different NSAIDs for its good pharmacological efficacy in clinical relief of primary dysmenorrhea. It is supposed that primary dysmenorrea is a disease associated with excessive uterine activity appearing during menstruation. In studied group of patients, the authors administered $10 \mathrm{mg}$ of piroxicam 1-2 hours before embryo transfer. The study showed that piroxicam increased the implantation rate in both fresh and frozen cycles. In fresh cycles, the implantation rate in the piroxicam group was $18.7 \%$ vs. $8,6 \%$ in controlled placebo group. In frozen cycles, the implantation rate in patients received piroxicam was also higher than in control group, (9.4\% and $2.3 \%$, respectively). The investigators observed greater beneficial effect of piroxicam in patients under 40 years of age in both fresh and frozen IVF cycles. The results confirmed the effectiveness of piroxicam in priming the uterus for embryo implantation.

Pierzyński et al. (Pierzyński et al.,2007) presented a case report describing the first administration of atosiban (oxytocin and vasopressin V1 receptor antagonist) in IVF treatment. The authors used intravenous bolus dose of $6.75 \mathrm{mg}$ of atosiban administered one hour before embryo transfer and continuing the intravenous infusion of atosiban solution up to 2 hours after the ET procedure. The total dose of administered drug was $37.5 \mathrm{mg}$. One hour before embryo transfer investigators assessed the patient's uterine activity using ultrasound, and detected mean 2.75 contractions per 1 minute during 4 minute ultrasound recording. Directly before embryo transfer, one hour after starting atosiban administration, the uterine contractility decreased to 1.75 contractions per 1 minute. Two good quality blastocyst were transferred to the uterus, without complications. Due to ethical reasons detecting the uterine contractility after 2 hours after ET, was not performed. The treatment resulted in twin pregnancy, delivered by caesarean section at the $29^{\text {th }}$ week of pregnancy because of vaginal bleeding suggesting the placental abruption. The previous preclinical 
study by the same authors did not present any toxic influence of atosiban on rabbit's embryo development and human sperm motility (Pierzyński et al.,2007).

Moraloglu et al. (Moraloglu et al.,2010) presented the first prospective, randomized, placebo-controlled clinical trial involving the administration the oxytocin antagonists before embryo transfer. 180 women undergoing intracytoplasmic sperm injection who had topquality embryos were randomly allocated into treatment and control groups. The studied patients received intravenous administration of atosiban before embryo transfer with a total administered dose of $3.75 \mathrm{mg}$. In the control group, the patients received placebo. The implantation rate per transfer in the atosiban patients was $20.4 \%$, which was significantly higher than in the control group $12.6 \%$. The miscarriage rates between studied group presented $16.7 \%$ in the atosiban patients group and $24.4 \%$ in the control group. These results indicated that atosiban increases the implantation rate after embryo transfer procedure. These results suggest that the treatment with the oxytocin antagonists before embryo transfer could be effective in priming of the uterus for implantation.

\section{The role of endometrial structure}

There are multiple studies that have supported or refuted the relationship between endometrial features such as thickness, length or pattern and the implantation rate (Bassil,2001; Kovacs et al.,2003; Richter et al.,2007; Zhang et al.,2005; Rinaldi et al.,1996). The data regarding the role of endometrial thickness as a predictor of implantation outcomes is still controversial. Most of the authors suggested that there is a trigger of endometrial thicknesses correlating with a significant reduction in the implantation rate (Bassil,2001; Weissman et al.,1999; Rinaldi et al.,1996). Some investigators tried to evaluate the endometrial pattern on the day of hCG administration, the day of oocyte retrieval or the day of embryo transfer and find some predicting markers of successful embryo implantation (Dickey et al.,1992; Rashidi et al.,2005), but the results did not show any statistically significant results. Fanchin et al. (Fanchin et al.,2000) proposed the assessment of the dynamics of endometrial growth to the triple layer together with endometrial thickness and endometrial echogenicity as prognostic markers in IVF treatment.

In IVF cycles, the prediction of the time of implantation window seems to be more difficult than in natural cycles. In stimulated cycles in comparison to natural, the excessive, nonphysiological hormones serum concentrations can be responsible for the different biochemical and biophysical changes in endometrium and can have different influence on embryo implantation. The endometrial changes in the days preceding the oocyte retrieval are important to clarify the impact of endometrial features in the prediction of a successful embryo implantation. The progression of the histological transformation of the endometrial layer and its receptivity can also differ depending on the type of the $\mathrm{COH}$ protocol and the doses and kinds of gonadotropins. Hormone dependant endometrial cell proliferation and differentiation seem to have an important value in endometrial receptivity and successful implantation (Bourgain et al.,2002; Bourgain,2004). The changes to endometrial echogenicity and structure are caused by rising progesterone serum levels and determine the implantation window (Fanchin et al.,2000; Bourgain et al.,2002).

The differences between endometrial dating in stimulated and natural cycles were presented in Bourgain et al. study (Bourgain et al.,2002). The rapid onset of secretory 
changes in endometrium was observed in IVF cycles on the day of the oocyte retrieval, corresponding to at least the second day after ovulation in natural cycles. These endometrial changes in IVF cycles were no longer visible after the second day after the oocyte retrieval and could be connected with the rapid increase of serum progesterone concentration appearing between the day of hCG administration and the day of oocyte retrieval. The implantation rate in IVF cycles seems to be not associated with progesterone serum levels, but progesterone can correlate with endometrial printing. The study showed that IVF cycles presented a significantly advanced endometrial maturation on the day of the oocyte retrieval when compared to natural cycles on the day of ovulation. No pregnancies were observed when the endometrial maturation was advanced for more than three days on the day of hCG administration.

Some authors (Kovacs et al.,2003; Richter et al.,2007) reported a significant increase of implantation rate observed with increase of endometrial thickness, independent from the patients' age and embryo quality. The mean endometrial thickness was greater in the cycles resulting in pregnancy than in the cycles not resulting in pregnancy $(11.9 \mathrm{~mm}$, and $11.3 \mathrm{~mm}$ respectively), but these differences were are not large. Authors also found a significant trend presenting a decrease in spontaneous miscarriages with an increase of endometrial thickness. Dickey et al. (Dickey et al.,1992) did not find significant differences in the pregnancy rate between patients with endometrial thickness of 9-13 $\mathrm{mm}$ and $14 \mathrm{~mm}$ assessed on the day of hCG administration. Weissman et al. (Weissman et al.,1999) suggested that the threshold of an endometrial thickness of $14-15 \mathrm{~mm}$ on the day of hCG administration a negative influence on implantation rates. Schild et al. (Schild et al.,2001) did not observed pregnancies in patients with an endometrial thickness of more than $15 \mathrm{~mm}$ on the day of embryo transfer, or more than $16 \mathrm{~mm}$ on the day of oocyte retrieval. Other investigators (Rinaldi et al.,1996) observed correlations between a minimal endometrial thickness of 9- $10 \mathrm{~mm}$ and higher implantation rates. Some authors (Kupesic et al.,2001; Schild et al.,2001) did not observe successful implantations when the endometrium was thinner than $7 \mathrm{~mm}$.

In the study by Kuć et al. (Kuć et al.,2011), relatively higher implantation rates were achieved if the endometrium thickness ranged between 12 and13 $\mathrm{mm}$ and at least one goodquality blastocyst was transferred in a double embryo transfer. The study showed that the endometrial thickness on the day of the triple layer appearance in the long GnRH agonist protocol and GnRH antagonist protocol was connected with a higher implantation rate. This was a first study analysing the dynamism of endometrial growth to triple layer in three different controlled ovarian stimulation protocols. The pregnancy rate in the GnRH antagonist protocol was statistically dependent on the time of the appearance of the triple layer as well as on the endometrial thickness on the day of the triple layer appearance. In the long GnRH agonist protocol only the endometrial thickness on the day of the triple layer appearance exerted an influence on the implantation rate. If the time between the first day of the appearance of the triple layer and the day of the hCG administration was longer than six days, no implantations were observed. The appearance of the triple layer after the $13^{\text {th }}$ day of stimulation was connected with the lack of implantations. The time of the appearance of the endometrial triple layer in $\mathrm{COH}$ cycles can be essential for endometrial dating and receptivity, as well as for the implantation window. 
The monitoring of patients on the day of hCG administration together with the day of ET could results in some useful information regarding successful implantation, than on any other day of treatment. The assessment of endometrial echogenicity performed at the end of the late follicular phase in IVF cycles was tested in search of a prognostic factor for embryo implantation (Giannaris et al.,2008; Richter et al.,2007; Schild et al.,2001). Some studies presented the hypothesis that endometrial features in the late follicular phase can predict the IVF outcomes, especially assessment of endometrial pattern and thickness (Scioscia et al.,2009; Giannaris et al.,2008; Richter et al.,2007; Zhang et al.,2005; Kovacs et al.,2003; Dietterich et al.,2002).
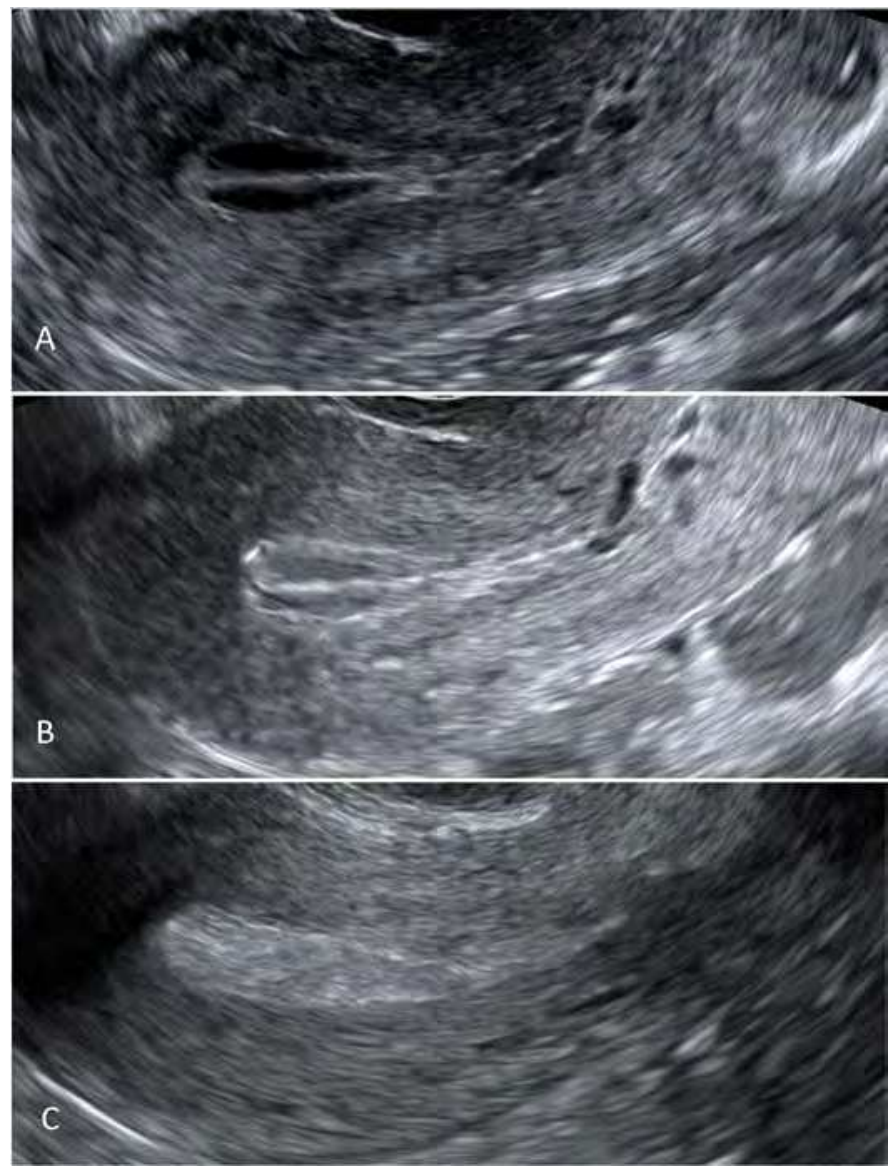

Fig. 10. Echogenicity of endometrial layer: A - hypoechogenic; B - isoechogenic; C hyperechogenic.

Some studies showed that the endometrial echogenicity (Figure 10) on the day of hCG administration or embryo transfer can be a predictive marker of IVF outcomes (Basir et al.,2002; Fanchin et al.,2000). Fanchin et al. (Fanchin et al.,2000) confirmed the influence of endometrial echogenicity on the implantation rate. High endometrial echogenicity assessed 
in ultrasound examination on the day of hCG administration was connected with the lower implantation rate, but in this study the endometrial echogenic pattern was not evaluated on the day of embryo transfer. The intensity of hyperechogenic transformation observed on the day of hCG administration may denote an acceleration of secretory changes in endometrium. Premature exposure of the endometrium to progesterone during the follicular phase in stimulated cycles can also lead to a faster progression of the endometrial echogenicity during the early luteal phase and can be associated with the lower implantation rate (Fanchin et al.,2000). The relationship between endometrial echogenicity observed during ultrasound examination and implantation rate was presented also in Kuć et al. study (Kuć et al.,2011). The hyperechogenic endometrium on the day of hCG administration was associated with the low pregnancy rate, but only in patients undergoing the long GnRH agonist protocol.

Some investigators oppose the role of endometrial echogenicity in predicting the successful implantation, and they do not find a positive correlation between endometrial thickness and endometrial pattern with implantation rate. The authors claimed that endometrial growth and its pattern during the controlled ovarian hyperstimulation did not influence on the implantation rate (Bassil,2001; Kupesic et al.,2001; Rashidi et al.,2005).

Zhang et al. (Zhang et al.,2005) showed a significant correlation between the endometrial thickness, the length of gonadotropins administration and their influence on implantation rates. Controlled ovarian hyperstimulation last longer than 11 days corresponded with thinner endometrium and lower implantation rate. Authors also suggested that in some groups of patients, the implantation rate can depend on the endometrial thickness, and the implantation rate was statistically unaffected by endometrial thickness when good-quality embryos were transferred.

Endometrial parameters could be helpful predictors of the successful implantation, but they should be analyzed in a multivariate analysis rather than used alone. The most precise evaluation of the impact that endometrial features have on the implantation rate should be carried out on patients with a single, high quality blastocyst transfer.

\section{Conclusions}

In conclusions, successful embryo implantation requires a genetically and morphologically healthy embryo as well as developed receptive endometrium. The interactions between the embryo and endometrial layer during implantation have been not still completely understood. Nowadays, most of investigators recommend single blastocyst transfer on the $5^{\text {th }}$ day after OR, if sufficient number of good quality embryos is cultured on the $3^{\text {rd }}$ day. Uterine contractions at the time of embryo transfer negatively affect embryo implantation, but the methods for assessing uterine activity are not sensitive enough. The constant progress in biomedical sciences allows in the future finding the new markers dating endometrial receptivity, and implantation window, as well as better selection of the cultured embryos.

\section{Acknowledgement}

The author would like to acknowledge Professor Waldemar Kuczyński MD PhD (Centre for Reproductive Medicine KRIOBANK, Białystok, Poland) for chapter revision support as well 
as Piotr Sieczyński MSc PhD (Centre for Reproductive Medicine KRIOBANK, Białystok, Poland) for help in editing the embryological images.

\section{References}

Alikani, M., Calderon, G., Tomkin, G., Garrisi, J., Kokot, M., \& Cohen, J. (2000). Cleavage anomalies in early human embryos and survival after prolonged culture in-vitro. Hum Reprod 15 (12):2634-43.

Ayoubi, J.M., Epiney, M., Brioschi, P.A., Fanchin, R., Chardonnens, D., \& de Ziegler, D. (2003). Comparison of changes in uterine contraction frequency after ovulation in the menstrual cycle and in in vitro fertilization cycles. Fertil Steril 79 (5):1101-5.

Balaban, B., Yakin, K., \& Urman, B. (2006). Randomized comparison of two different blastocyst grading systems. Fertil Steril 85 (3):559-63.

Basir, G.S., O, W.S., So, W.W., Ng, E.H., \& Ho, P.C. (2002). Evaluation of cycle-to-cycle variation of endometrial responsiveness using transvaginal sonography in women undergoing assisted reproduction. Ultrasound Obstet Gynecol 19 (5):484-9.

Bassil, S. (2001). Changes in endometrial thickness, width, length and pattern in predicting pregnancy outcome during ovarian stimulation in in vitro fertilization. Ultrasound Obstet Gynecol 18 (3):258-63.

Bernabeu, R., Roca, M., Torres, A., \& Ten, J. (2006). Indomethacin effect on implantation rates in oocyte recipients. Hum Reprod 21 (2):364-9.

Blake, D.A., Farquhar, C.M., Johnson, N., \& Proctor, M. (2007). Cleavage stage versus blastocyst stage embryo transfer in assisted conception. Cochrane Database Syst Rev (4):CD002118.

Bourgain, C. (2004). [Endometrial biopsy in the evaluation of endometrial receptivity]. J Gynecol Obstet Biol Reprod (Paris) 33 (1 Pt 2):S13-7.

Bourgain, C., Ubaldi, F., Tavaniotou, A., Smitz, J., Van Steirteghem, A.C., \& Devroey, P. (2002). Endometrial hormone receptors and proliferation index in the periovulatory phase of stimulated embryo transfer cycles in comparison with natural cycles and relation to clinical pregnancy outcome. Fertil Steril 78 (2):237-44.

Brezinova, J., Oborna, I., Svobodova, M., \& Fingerova, H. (2009). Evaluation of day one embryo quality and IVF outcome--a comparison of two scoring systems. Reprod Biol Endocrinol 7:9.

Bulletti, C., \& de Ziegler, D. (2005). Uterine contractility and embryo implantation. Curr Opin Obstet Gynecol 17 (3):265-76.

Bulletti, C., \& de Ziegler, D. (2006). Uterine contractility and embryo implantation. Curr Opin Obstet Gynecol 18 (4):473-84.

Carrillo, A.J., Lane, B., Pridman, D.D., Risch, P.P., Pool, T.B., Silverman, I.H., \& Cook, C.L. (1998). Improved clinical outcomes for in vitro fertilization with delay of embryo transfer from 48 to 72 hours after oocyte retrieval: use of glucose- and phosphatefree media. Fertil Steril 69 (2):329-34.

Charnock-Jones, D.S., Sharkey, A.M., Boocock, C.A., Ahmed, A., Plevin, R., Ferrara, N., \& Smith, S.K. (1994). Vascular endothelial growth factor receptor localization and activation in human trophoblast and choriocarcinoma cells. Biol Reprod 51 (3):524-30.

Ciray, H.N., Ulug, U., \& Bahceci, M. (2004). Transfer of early-cleaved embryos increases implantation rate in patients undergoing ovarian stimulation and ICSI-embryo transfer. Reprod Biomed Online 8 (2):219-23. 
Ciray, N. (2007). Even early cleavage and day 2 embryo score. Reprod Biomed Online 14 (5):666; author reply 666.

Desai, N.N., Goldstein, J., Rowland, D.Y., \& Goldfarb, J.M. (2000). Morphological evaluation of human embryos and derivation of an embryo quality scoring system specific for day 3 embryos: a preliminary study. Hum Reprod 15 (10):2190-6.

Dickey, R.P., Olar, T.T., Curole, D.N., Taylor, S.N., \& Rye, P.H. (1992). Endometrial pattern and thickness associated with pregnancy outcome after assisted reproduction technologies. Hum Reprod 7 (3):418-21.

Dietterich, C., Check, J.H., Choe, J.K., Nazari, A., \& Lurie, D. (2002). Increased endometrial thickness on the day of human chorionic gonadotropin injection does not adversely affect pregnancy or implantation rates following in vitro fertilization-embryo transfer. Fertil Steril 77 (4):781-6.

Dokras, A., Sargent, I.L., \& Barlow, D.H. (1993). Human blastocyst grading: an indicator of developmental potential? Hum Reprod 8 (12):2119-27.

Fanchin, R., Ayoubi, J.M., Righini, C., Olivennes, F., Schonauer, L.M., \& Frydman, R. (2001). Uterine contractility decreases at the time of blastocyst transfers. Hum Reprod 16 (6):1115-9.

Fanchin, R., Righini, C., Ayoubi, J.M., Olivennes, F., de Ziegler, D., \& Frydman, R. (2000). New look at endometrial echogenicity: objective computer-assisted measurements predict endometrial receptivity in in vitro fertilization-embryo transfer. Fertil Steril 74 (2):274-81.

Fanchin, R., Righini, C., Olivennes, F., Taylor, S., de Ziegler, D., \& Frydman, R. (1998). Uterine contractions at the time of embryo transfer alter pregnancy rates after invitro fertilization. Hum Reprod 13 (7):1968-74.

Fanchin, R., Righini, C., Schonauer, L.M., Olivennes, F., Cunha Filho, J.S., \& Frydman, R. (2001). Vaginal versus oral E(2) administration: effects on endometrial thickness, uterine perfusion, and contractility. Fertil Steril 76 (5):994-8.

Fauque, P., Leandri, R., Merlet, F., Juillard, J.C., Epelboin, S., Guibert, J., Jouannet, P., \& Patrat, C. (2007). Pregnancy outcome and live birth after IVF and ICSI according to embryo quality. J Assist Reprod Genet 24 (5):159-65.

Gardner, D.K., Lane, M., Stevens, J., Schlenker, T., \& Schoolcraft, W.B. (2000). Blastocyst score affects implantation and pregnancy outcome: towards a single blastocyst transfer. Fertil Steril 73 (6):1155-8.

Giannaris, D., Zourla, A., Chrelias, C., Loghis, C., \& Kassanos, D. (2008). Ultrasound assessment of endometrial thickness: correlation with ovarian stimulation and pregnancy rates in IVF cycles. Clin Exp Obstet Gynecol 35 (3):190-3.

Giorgetti, C., Hans, E., Terriou, P., Salzmann, J., Barry, B., Chabert-Orsini, V., Chinchole, J.M., Franquebalme, J.P., Glowaczower, E., Sitri, M.C., Thibault, M.C., \& Roulier, R. (2007). Early cleavage: an additional predictor of high implantation rate following elective single embryo transfer. Reprod Biomed Online 14 (1):85-91.

Giudice, L. 2003. Implantation and endometrial function. In Reproductive medicine: mollecular, cellular, and genetic fundaments. , edited by B. F: Boca Roton: Parthenon Pub Group.

Kallen, B., Finnstrom, O., Lindam, A., Nilsson, E., Nygren, K.G., \& Olausson, P.O. (2010). Blastocyst versus cleavage stage transfer in in vitro fertilization: differences in neonatal outcome? Fertil Steril 94 (5):1680-3. 
Kawachiya, S., Bodri, D., Shimada, N., Kato, K., Takehara, Y., \& Kato, O. (2011). Blastocyst culture is associated with an elevated incidence of monozygotic twinning after single embryo transfer. Fertil Steril 95 (6):2140-2.

Kissler, S., Siebzehnruebl, E., Kohl, J., Mueller, A., Hamscho, N., Gaetje, R., Ahr, A., Rody, A., \& Kaufmann, M. (2004). Uterine contractility and directed sperm transport assessed by hysterosalpingoscintigraphy (HSSG) and intrauterine pressure (IUP) measurement. Acta Obstet Gynecol Scand 83 (4):369-74.

Kitlas, A., Oczeretko, E., Swiatecka, J., Borowska, M., \& Laudanski, T. (2009). Uterine contraction signals--application of the linear synchronization measures. Eur J Obstet Gynecol Reprod Biol 144 Suppl 1:S61-4.

Kovacs, P., Matyas, S., Boda, K., \& Kaali, S.G. (2003). The effect of endometrial thickness on IVF/ICSI outcome. Hum Reprod 18 (11):2337-41.

Kuć, P., Kuczyńska, A., Topczewska, M., Tadejko, P., \& Kuczyński, W. (2011). The dynamics of endometrial growth and the triple layer appearance in three different controlled ovarian hyperstimulation protocols and their influence on IVF outcomes. Gynecol Endocrinol 27 (11): 867-873.

Kupesic, S., Bekavac, I., Bjelos, D., \& Kurjak, A. (2001). Assessment of endometrial receptivity by transvaginal color Doppler and three-dimensional power Doppler ultrasonography in patients undergoing in vitro fertilization procedures. $J$ Ultrasound Med 20 (2):125-34.

Ludwig, M., Schopper, B., Katalinic, A., Sturm, R., Al-Hasani, S., \& Diedrich, K. (2000). Experience with the elective transfer of two embryos under the conditions of the german embryo protection law: results of a retrospective data analysis of 2573 transfer cycles. Hum Reprod 15 (2):319-24.

Moon, H.S., Park, S.H., Lee, J.O., Kim, K.S., \& Joo, B.S. (2004). Treatment with piroxicam before embryo transfer increases the pregnancy rate after in vitro fertilization and embryo transfer. Fertil Steril 82 (4):816-20.

Moraloglu, O., Tonguc, E., Var, T., Zeyrek, T., \& Batioglu, S. (2010). Treatment with oxytocin antagonists before embryo transfer may increase implantation rates after IVF. Reprod Biomed Online 21 (3):338-43.

Paiva, P., Salamonsen, L.A., Manuelpillai, U., Walker, C., Tapia, A., Wallace, E.M., \& Dimitriadis, E. (2007). Interleukin-11 promotes migration, but not proliferation, of human trophoblast cells, implying a role in placentation. Endocrinology 148 (11):5566-72.

Papanikolaou, E.G., Camus, M., Kolibianakis, E.M., Van Landuyt, L., Van Steirteghem, A., \& Devroey, P. (2006). In vitro fertilization with single blastocyst-stage versus single cleavage-stage embryos. N Engl J Med 354 (11):1139-46.

Papanikolaou, E.G., Fatemi, H., Venetis, C., Donoso, P., Kolibianakis, E., Tournaye, H., Tarlatzis, B., \& Devroey, P. (2010). Monozygotic twinning is not increased after single blastocyst transfer compared with single cleavage-stage embryo transfer. Fertil Steril 93 (2):592-7.

Pierzyński, P., Gajda, B., Smorag, Z., Rasmussen, A.D., \& Kuczyński, W. (2007). Effect of atosiban on rabbit embryo development and human sperm motility. Fertil Steril 87 (5):1147-52.

Pierzyński, P., Reinheimer, T.M., \& Kuczyński, W. (2007). Oxytocin antagonists may improve infertility treatment. Fertil Steril 88 (1):213 e19-22. 
Poindexter, A.N., 3rd, Thompson, D.J., Gibbons, W.E., Findley, W.E., Dodson, M.G., \& Young, R.L. (1986). Residual embryos in failed embryo transfer. Fertil Steril 46 (2):262-7.

Rabotti, C., Mischi, M., van Laar, J.O., Oei, S.G., \& Bergmans, J.W. (2009). Myometrium electromechanical modeling for internal uterine pressure estimation by electrohysterography. Conf Proc IEEE Eng Med Biol Soc 2009:6259-62.

Racowsky, C., Combelles, C.M., Nureddin, A., Pan, Y., Finn, A., Miles, L., Gale, S., O'Leary, T., \& Jackson, K.V. (2003). Day 3 and day 5 morphological predictors of embryo viability. Reprod Biomed Online 6 (3):323-31.

Rashidi, B.H., Sadeghi, M., Jafarabadi, M., \& Tehrani Nejad, E.S. (2005). Relationships between pregnancy rates following in vitro fertilization or intracytoplasmic sperm injection and endometrial thickness and pattern. Eur J Obstet Gynecol Reprod Biol 120 (2):179-84.

Rehman, K.S., Bukulmez, O., Langley, M., Carr, B.R., Nackley, A.C., Doody, K.M., \& Doody, K.J. (2007). Late stages of embryo progression are a much better predictor of clinical pregnancy than early cleavage in intracytoplasmic sperm injection and in vitro fertilization cycles with blastocyst-stage transfer. Fertil Steril 87 (5):1041-52.

Richter, K.S., Bugge, K.R., Bromer, J.G., \& Levy, M.J. (2007). Relationship between endometrial thickness and embryo implantation, based on 1,294 cycles of in vitro fertilization with transfer of two blastocyst-stage embryos. Fertil Steril 87 (1):53-9.

Rinaldi, L., Lisi, F., Floccari, A., Lisi, R., Pepe, G., \& Fishel, S. (1996). Endometrial thickness as a predictor of pregnancy after in-vitro fertilization but not after intracytoplasmic sperm injection. Hum Reprod 11 (7):1538-41.

Saldeen, P., \& Sundstrom, P. (2005). Nuclear status of four-cell preembryos predicts implantation potential in in vitro fertilization treatment cycles. Fertil Steril 84 (3):584-9.

Schild, R.L., Knobloch, C., Dorn, C., Fimmers, R., van der Ven, H., \& Hansmann, M. (2001). Endometrial receptivity in an in vitro fertilization program as assessed by spiral artery blood flow, endometrial thickness, endometrial volume, and uterine artery blood flow. Fertil Steril 75 (2):361-6.

Scioscia, M., Lamanna, G., Lorusso, F., Serrati, G., Selvaggi, L.E., \& Depalo, R. (2009). Characterization of endometrial growth in proliferative and early luteal phase in IVF cycles. Reprod Biomed Online 18 (1):73-8.

Scott, L. (2003). Pronuclear score as a predictor of embryo development. Reprod Biomed Online 6:201-214.

Scott, L., Alvero, R., Leondires, M., \& Miller, B. (2000). The morphology of human pronuclear embryos is positively related to blastocyst development and implantation. Hum Reprod 15 (11):2394-403.

Scott, L.A., \& Smith, S. (1998). The successful use of pronuclear embryo transfers the day following oocyte retrieval. Hum Reprod 13 (4):1003-13.

Simon, C., Gimeno, M.J., Mercader, A., Frances, A., Garcia Velasco, J., Remohi, J., Polan, M.L., \& Pellicer, A. (1996). Cytokines-adhesion molecules-invasive proteinases. The missing paracrine/autocrine link in embryonic implantation? Mol Hum Reprod 2 (6):405-24.

Skiadas, C.C., Jackson, K.V., \& Racowsky, C. (2006). Early compaction on day 3 may be associated with increased implantation potential. Fertil Steril 86 (5):1386-91. 
Terriou, P., Giorgetti, C., Hans, E., Salzmann, J., Charles, O., Cignetti, L., Avon, C., \& Roulier, R. (2007). Relationship between even early cleavage and day 2 embryo score and assessment of their predictive value for pregnancy. Reprod Biomed Online 14 (3):294-9.

Terriou, P., Sapin, C., Giorgetti, C., Hans, E., Spach, J.L., \& Roulier, R. (2001). Embryo score is a better predictor of pregnancy than the number of transferred embryos or female age. Fertil Steril 75 (3):525-31.

Tesarik, J., \& Greco, E. (1999). The probability of abnormal preimplantation development can be predicted by a single static observation on pronuclear stage morphology. Hum Reprod 14 (5):1318-23.

Weissman, A., Gotlieb, L., \& Casper, R.F. (1999). The detrimental effect of increased endometrial thickness on implantation and pregnancy rates and outcome in an in vitro fertilization program. Fertil Steril 71 (1):147-9.

White, C.A., Dimitriadis, E., Sharkey, A.M., Stoikos, C.J., \& Salamonsen, L.A. (2007). Interleukin 1 beta is induced by interleukin 11 during decidualization of human endometrial stromal cells, but is not released in a bioactive form. J Reprod Immunol 73 (1):28-38.

Zander-Fox, D.L., Tremellen, K., \& Lane, M. (2011). Single blastocyst embryo transfer maintains comparable pregnancy rates to double cleavage-stage embryo transfer but results in healthier pregnancy outcomes. Aust N Z J Obstet Gynaecol.

Zhang, X., Chen, C.H., Confino, E., Barnes, R., Milad, M., \& Kazer, R.R. (2005). Increased endometrial thickness is associated with improved treatment outcome for selected patients undergoing in vitro fertilization-embryo transfer. Fertil Steril 83 (2):336-40.

Zollner, U., Zollner, K.P., Hartl, G., Dietl, J., \& Steck, T. (2002). The use of a detailed zygote score after IVF/ICSI to obtain good quality blastocysts: the German experience. Hum Reprod 17 (5):1327-33. 


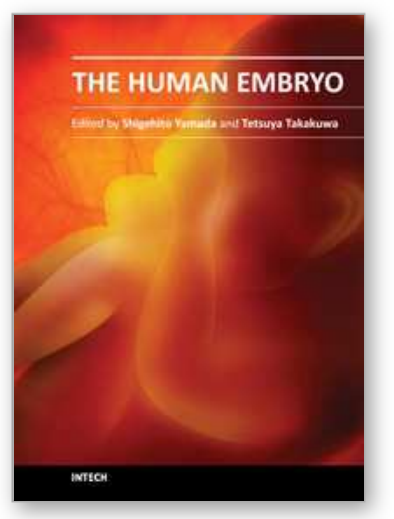

\author{
The Human Embryo \\ Edited by Dr. Shigehito Yamada
}

ISBN 978-953-51-0124-6

Hard cover, 180 pages

Publisher InTech

Published online 02, March, 2012

Published in print edition March, 2012

Human embryology is now rapidly moving to a new phase due to recent innovation and advances of life science including ES and iPS technology. This new era also directs a difficult challenge for scientists in terms of technological and ethical issues for future human embryology. However, human embryology is difficult to research due to ethics involved in the collection of human materials. This book traces the early history and provides knowledge on demonstration of principles from ancient to the most recent embryo studies amidst the unresolved scientific and ethical issues. We hope this book will help the readers to understand human embryo development better.

\title{
How to reference
}

In order to correctly reference this scholarly work, feel free to copy and paste the following:

Paweł Kuć (2012). Optimal Environment for the Implantation of Human Embryo, The Human Embryo, Dr. Shigehito Yamada (Ed.), ISBN: 978-953-51-0124-6, InTech, Available from:

http://www.intechopen.com/books/the-human-embryo/optimal-environment-for-the-implantation-of-humanembryo-

\section{INTECH}

open science | open minds

\section{InTech Europe}

University Campus STeP Ri Slavka Krautzeka 83/A 51000 Rijeka, Croatia

Phone: +385 (51) 770447

Fax: +385 (51) 686166 www.intechopen.com

\section{InTech China}

Unit 405, Office Block, Hotel Equatorial Shanghai No.65, Yan An Road (West), Shanghai, 200040, China 中国上海市延安西路65号上海国际贵都大饭店办公楼405单元 Phone: +86-21-62489820

Fax: $+86-21-62489821$ 
(C) 2012 The Author(s). Licensee IntechOpen. This is an open access article distributed under the terms of the Creative Commons Attribution 3.0 License, which permits unrestricted use, distribution, and reproduction in any medium, provided the original work is properly cited. 